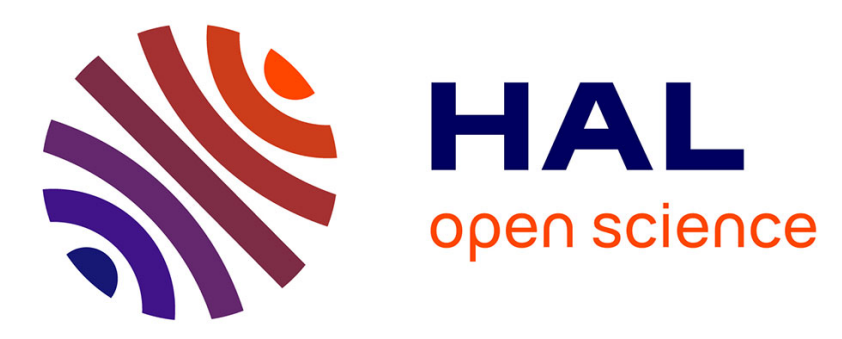

\title{
Modeling the climb-assisted glide of edge dislocations through a random distribution of nanosized vacancy clusters
}

\author{
Marie Landeiro dos Reis, Laurent Proville, Maxime Sauzay
}

\section{- To cite this version:}

Marie Landeiro dos Reis, Laurent Proville, Maxime Sauzay. Modeling the climb-assisted glide of edge dislocations through a random distribution of nanosized vacancy clusters. Physical Review Materials, 2018, 2, pp.093604. 10.1103/PhysRevMaterials.2.093604 . cea-02447261

HAL Id: cea-02447261

https://hal-cea.archives-ouvertes.fr/cea-02447261

Submitted on 21 Jan 2020

HAL is a multi-disciplinary open access archive for the deposit and dissemination of scientific research documents, whether they are published or not. The documents may come from teaching and research institutions in France or abroad, or from public or private research centers.
L'archive ouverte pluridisciplinaire HAL, est destinée au dépôt et à la diffusion de documents scientifiques de niveau recherche, publiés ou non, émanant des établissements d'enseignement et de recherche français ou étrangers, des laboratoires publics ou privés. 


\title{
Modeling the climb-assisted glide of edge dislocations through a random distribution of nanosized vacancy clusters
}

\author{
Marie Landeiro Dos Reis* and Laurent Proville \\ DEN-Service de Recherches de Métallurgie Physique, CEA, Université Paris-Saclay, F-91191, Gif-sur-Yvette, France \\ Maxime Sauzay \\ DEN-Service de Recherches de Métallurgie Appliquée, CEA, Université Paris-Saclay, F-91191, Gif-sur-Yvette, France
}

(Received 14 June 2018; published 10 September 2018)

\begin{abstract}
A multiscale model is developed to simulate the climb-assisted glide of edge dislocations anchored by a random distribution of nanosized vacancy clusters. Atomic-scale simulations allowed us to characterize the interactions between an edge dislocation and nanovoids as a function of their sizes and shapes. The atomic-scale data were used to calibrate the parameters of an elastic line model, which we employed to evaluate the average glide distance of a dislocation with realistic dimensions. To complete our scheme, a standard model for the climb velocity of edge dislocations was enhanced with atomic-scale inputs in order to determine the deformation rate expected through the climb-assisted glide. Our predictions made for the archetypical case of Al are in good agreement with experiments of different types, i.e., tensile deformation tests and steady creep tests.
\end{abstract}

DOI: 10.1103/PhysRevMaterials.2.093604

\section{INTRODUCTION}

According to direct observations through transmission electronic microscopy (TEM) in various face centered cubic (fcc) metals, vacancy clusters may form along the processes of cold deformation or quenching (see papers of Kiritani [1,2], Kojima et al. [3], and Loretto et al. [4,5]). Dai and Victoria [6] observed these defects in a single crystal of $\mathrm{Cu}$, where they show that their density increases with strain. In parallel, molecular dynamic studies, carried out by Wang et al. [7-11] and Brinckmann et al. [12] confirmed that the vacancy clusters can be formed by nonscrew dislocation annihilations, a clue suggesting that some nanovoids might continually form during the deformation process, as expected earlier by Saada [13]. At room temperature, vacancy clusters seem to take the shape of Stacking-Fault Tetraedra (SFT) $[1,3,4,6]$ with a typical size of $2.5 \mathrm{~nm}$, in average [6]. After quenching from very high temperatures, some faceted voids were also observed in $\mathrm{Al}$ [1]. In course of a deformation process, the vacancy clusters anchor the dislocations and therefore they could contribute to the hardening of materials. Under the combined effects of thermal activation and applied shear stress, the dislocations may eventually pass the obstacles by simple glide [14-18] or by cross-slip for screw-type dislocations $[19,20]$. However, when the applied shear stress is much smaller than the critical stress to glide or to cross-slip, the diffusion of vacancies is possible at sufficiently high temperatures. Then the climb of edge dislocations becomes efficient and these dislocations may circumvent the obstacles perpendicularly to their glide plane, before they glide until they encounter another pinning configuration [21-26]. Such a process was proposed by Weertman to explain the stress exponent of the deformation rate in the

\footnotetext{
*marie.landeirodosreis@cea.fr
}

steady creep [27]. Therefore, provided that voids could exist in a certain range of temperature [28,29], the deformation of an fcc crystal is expected to proceed through the glide of edge dislocations assisted by climb. In order to predict the strain rate $\dot{\gamma}$ associated with such a process, we constructed a model made of three components, treating the different space and time scales that are involved in the climb-assisted glide: (i) atomicscale simulations allowed us to characterize the interaction between dislocations and the vacancy clusters. They were used also to determine the formation of jogs along the climb of an anchored dislocation. (ii) A two-dimensional elastic line model [30-34] is employed to determine the typical glide distance of dislocations, denoted by $\bar{d}$. The parameters of such a model were calibrated according to our atomic-scale simulations. (iii) Finally, we used the standard theory of dislocations [35] to determine the time required for dislocation climb $t_{\text {climb }}$, which allowed us to complete the Orowan equation and to fix the strain rate as $\dot{\gamma}=\rho b \bar{d} / t_{\text {climb }}$, where $\rho$ stands for the dislocation density and $b$ is the norm of the dislocation Burgers vector. The predictions of the theory agree well with experimental data either from tensile deformation tests [36] or from steady-state creep (SSC) experiments [37-40]. SSC is a complex process to which multiple mechanisms may contribute concurrently and many propositions were made about its theoretical frame [41-52]. Although the presence of vacancy clusters was not confirmed in SSC experiments, we think that our study could motivate some interest about the possibility of such obstacles, in addition to the more standard dislocation forest [21,22,27]. Furthermore, the mechanism of glide assisted by climb could be of some importance in more complex materials such as steels, where the obstacles could be formed by impurities and precipitates [53], instead of vacancy clusters. In the present work, the activity of dislocation sources as well as the interaction between the dislocations were not accounted for. To that respect, simulations based on discrete the dislocations 
dynamics method $[21,22,54]$ or continuous mean-field theory [55] are more appropriate. Our study is concentrated on an essential parameter needed in such methods, i.e., the single dislocation mobility.

The present paper is organized as follows. The simulation methods are detailed in Sec. II and our results are presented and discussed in Sec. III. In Sec. IV, we summarize our work and draw some perspectives.

\section{SIMULATION METHOD}

\section{A. Interaction between a nanovoid and a dislocation}

Molecular static simulations were employed to determine the interaction between a single edge dislocation and a vacancy cluster in $\mathrm{Al}$. The interatomic forces were computed from the embedded atom method (EAM), which allowed us to treat large simulation cells in comparison to more accurate methods based on the density functional theory [56]. We used an EAM version proposed by Liu et al. [57] for a potential developed originally by Ercolessi et al. [58]. Such a potential is commonly used to study dislocations because of its robustness in that field (see Refs. [16,59] for the proper validation of the potential). The simulation cell involves 750000 atoms and its orientation is [110] along $\mathrm{X},[\overline{1} 12]$ along $\mathrm{Y}$, and [1111] along $\mathrm{Z}$ [see Fig. 1(a)]. The size of the simulation cell has been determined to avoid artifacts due to boundary conditions. The atoms are placed according to the fcc symmetry. We performed simulations with periodic boundary conditions in the $\mathrm{X}$ and $\mathrm{Y}$ directions. In order to study the dislocation motion under an applied shear stress, we introduced free surfaces orthogonal to $\mathrm{Z}$. The details of the simulation cell and the introduction of an edge dislocation are detailed in Refs. [20,34]. The dislocation Burgers vector is $\frac{\mathrm{a}_{0}}{2}[110]$ where $a_{0}$ corresponds to the lattice parameter of the perfect fcc crystal. The norm of the Burgers vector is $b=a_{0} / \sqrt{2}$. In order to add a spherical cavity, we merely removed atoms located at a distance less than $r$, from a selected position in the crystal. Then the atomic positions were relaxed by adding a Langevin damping into the dynamical equation of motion. The latter was integrated with a standard velocity VERLET algorithm [60]. The convergence criterion on the interatomic forces was $10^{-5} \mathrm{eV} / \AA$. To apply a stress on the free surfaces, we added constant forces on each atom located near these surfaces [20]. At each stress increment $(<1 \mathrm{MPa})$, the relaxation was repeated with the same convergence criterion. Increasing gradually the shear stress component $\tau$, corresponding to the orientation [110]\{111\}, we determined the critical shear stress $\tau_{\mathrm{c}}$ allowing the dislocation to pass the cavity [61]. An example of a dislocation anchored by a cavity is presented in Fig. 1(a), where the atoms were colored according to the configuration of their first neighbor cell.

The pinning force $f_{p}$ of vacancy clusters was determined through the balance with the Peach-Koehler force:

$$
f_{p}=\tau_{c} b L,
$$

where $\tau_{c}$ is the minimum shear stress required to unpin the dislocation from the void and $\mathrm{L}$ is the length of the dislocation line in the Y direction, i.e., the dimension of the simulation cell. From the atomic-scale simulations, we computed $\tau_{c}$ for
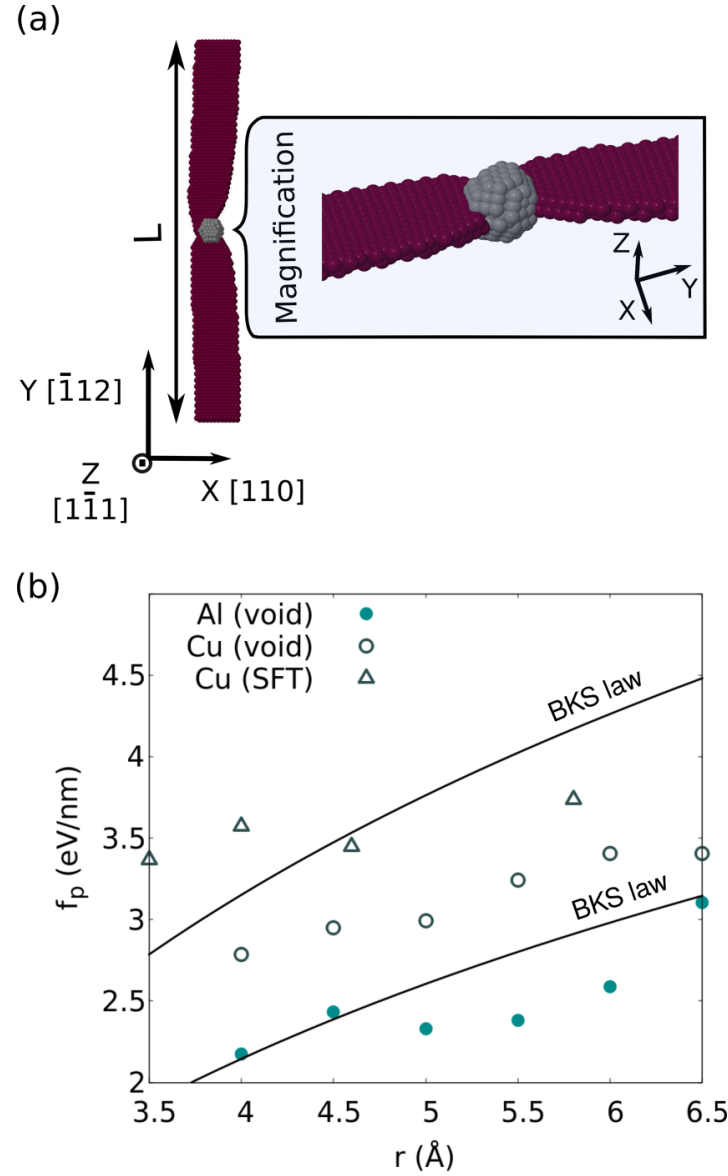

FIG. 1. (a) 3D view of our simulation cell where we have selected atoms situated in the stacking fault ribbon of an edge dislocation (purple), pinned by a nanovoid (gray). (b) Pinning force $f_{p}$ against the cluster size in $\mathrm{Al}$ and $\mathrm{Cu}$, for different shape of clusters (spherical voids and SFT), computed in molecular static simulations (symbols) and from BKS theory $[63,64]$ (lines), see Eq. (3). The simulation size in the $\mathrm{Y}$ direction, noticed by $\mathrm{L}$, is $26 \mathrm{~nm}$ for $\mathrm{Cu}$, and $29 \mathrm{~nm}$ for $\mathrm{Al}$.

voids with different radius $r$ (see Table I) in order to obtain a relation between $f_{p}$ and $r$. We followed the same procedure as Ref. [61]. For comparison, we performed the same calculations in the case of $\mathrm{Cu}$, using the EAM developed by Mishin et al. [62], with the same simulation cell geometry as for Al. As expected, the results reported in Fig. 1(b) show that the larger the cluster the stronger the pinning force $f_{p}$. Our computations show a dependence of $f_{p}$ on the cluster shape. We determined the effective radius $r$ for SFT by counting the number of vacancies $n_{v}$ in the cluster and equating it to the number vacancies in a spherical void of radius $r$. This yields the formula $n_{v}=\frac{4}{3} \pi r^{3} / V_{\text {at }}$, where $V_{\text {at }}$ is the atomic volume.

TABLE I. Critical stress $\tau_{c}$ to unpin an edge dislocation from a cavity of radius $r$. Activation energy $E_{\text {act }}$ for the same process under zero stress (see Fig. 2).

\begin{tabular}{lcccccc}
\hline \hline$r(\AA)=$ & 4 & 4.5 & 5 & 5.5 & 6 & 6.5 \\
\hline$\tau_{c}(\mathrm{MPa})$ & 42 & 47 & 45 & 46 & 50 & 60 \\
$E_{\text {act }}(\mathrm{eV})$ & & 2.37 & 2.32 & 2.79 & 2.82 & 3.70 \\
\hline \hline
\end{tabular}


Bacon, Kocks, and Scattergood (BKS) [63,64] derived an analytical formula for $\tau_{c}$, which is written as follows:

$$
\tau_{\mathrm{BKS}}=\frac{\mu_{0} b}{2 \pi L}\left[\ln \left(\frac{2 r L}{(2 r+L) b}\right)+B\right],
$$

where $B$ is a parameter adjusted to fit the simulation data, $r$ is the vacancy cluster radius, and $\mu_{0}$ is the shear modulus equal to $25.4 \mathrm{GPa}$ for $\mathrm{Al}$ and $42.1 \mathrm{GPa}$ for $\mathrm{Cu}$ [65]. For both $\mathrm{Al}$ and $\mathrm{Cu}$, the adjustment of $B$ against our atomic-scale simulations yields $B=0.01$ [see Fig. 1(b)]. The logarithmic variation predicted by BKS agrees rather well with the atomic-scale simulations. Combining Eqs. (1) and (2), we obtained for $2 r \ll L$,

$$
f_{p}=\frac{\mu_{0} b^{2}}{2 \pi}\left[\ln \left(\frac{2 r}{b}\right)+B\right] .
$$

This unequivocal relation between the obstacle size $r$ and the pinning force $f_{p}$ is used to fix the pinning strength of obstacles in the elastic line model presented in the following section. The difference in $f_{p}$ obtained from simulations for voids and for SFTs [see Fig. 1(b)] is not described in BKS theory, so we assumed that it is negligible for clusters of sizes comparable to those observed experimentally.

In order to compute the interaction potential between the cavity and the dislocation, we used the nudged elastic band method (NEB) $[66,67]$ with a constant spring force of $k_{\mathrm{NEB}}=$ $10 \mathrm{eV} / \AA$ and 130 NEB images. The starting configuration for each image was determined previously from the molecular quasistatic simulations realized at a stress level above the critical shear stress. Since the stress was sufficiently large to permit the dislocation to pass the obstacle, we recorded regularly the atomic configurations along the dislocation trajectory and we used them as starting configurations for the NEB. The relaxation in NEB was performed until the maximum interatomic force becomes less than $10^{-4} \mathrm{eV} / \AA$, which allows us to determine the minimum energy path (MEP). The MEPs associated to different cavity radii $r$ are presented in Fig. 2(a). We noticed that the interaction between a dislocation and a vacancy cluster is attractive. When the dislocation crosses the cavity, the latter is sheared and a step corresponding to one Burgers vector is formed as shown in Fig. 2(b). Hence the initial and the final energy along the MEP are not equal. We noticed that the larger is the cavity the larger is the difference between the initial and the final energies. The difference in energy between the final state and the bottom of the MEP [see Fig. 2(a)] corresponds to the thermal energy required to unpin the dislocation, denoted by $E_{\text {act }}$. The mechanical work required to cross a cavity can be approximated by the product between the pinning force $f_{P}$ and the distance that the dislocation has to progress in order to cross the cavity. The latter is less than $2 r$. Therefore one deduces that $E_{\text {act }} \approx 2 r f_{p}$, where $\mathrm{f}_{\mathrm{p}}$ is given by Eq. (3). The simulation data obtained by the NEB method were reported in Table I and they were plotted in Fig. 2(c) along with the analytical approximation $E_{\text {act }}=2 r f_{p}$. A quite good agreement was obtained, therefore showing that the BKS theory can be used to estimate the height of the potential energy barrier that corresponds to the dislocation unpinning from a nanovoid.

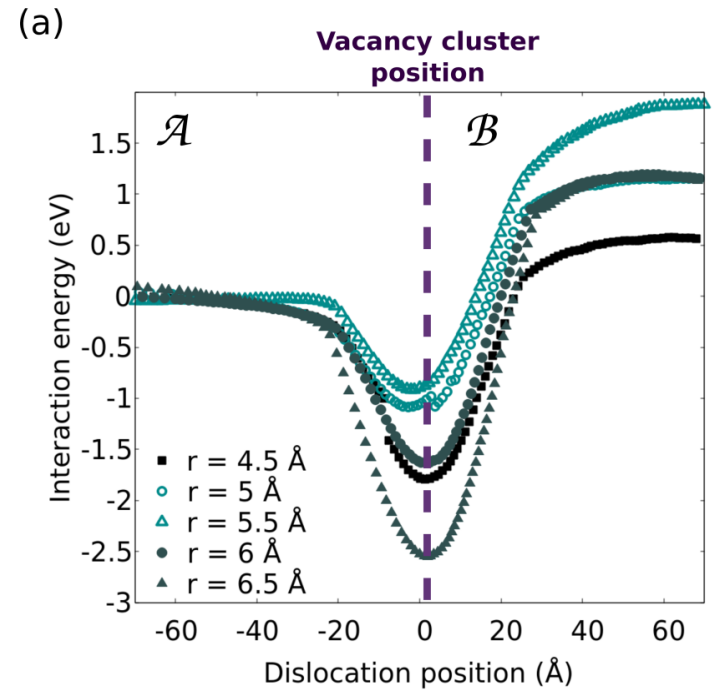

(b)

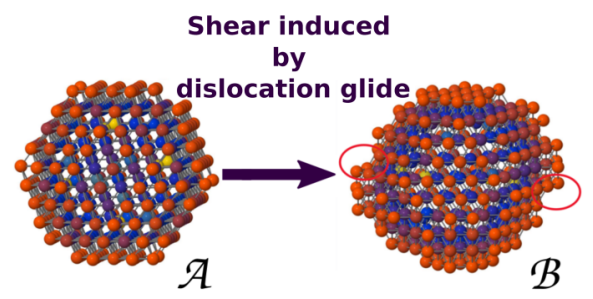

(c)

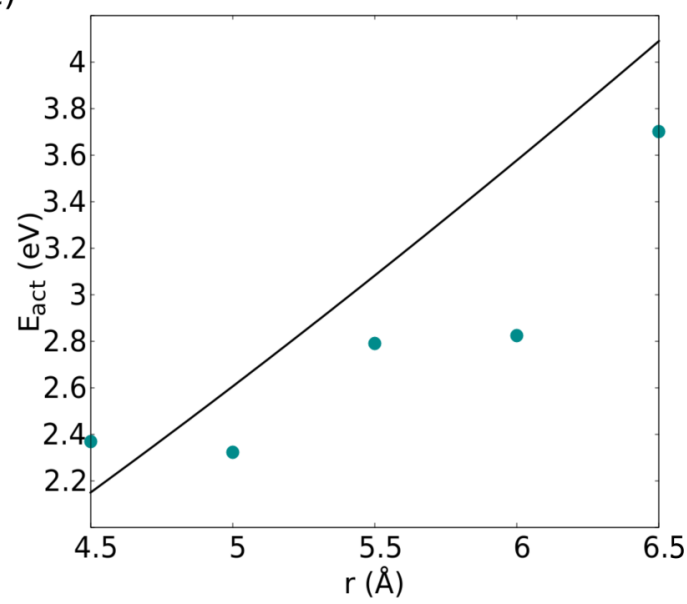

FIG. 2. (a) Potential energy of the simulation cell against the position of the dislocation bypassing a vacancy cluster of radius $r$, computed from the NEB method [66,67] with no applied stress. Each symbol corresponds to a NEB image. (b) Cavity before $(\mathcal{A})$ and after $(\mathcal{B})$ the dislocation crossing. The upper and the lower parts of the cavity were shifted by one Burgers vector with respect to each other, thereby forming steps indicated by red circles. (c) Variation of the energy barrier for a dislocation crossing a nanovoid of radius $r$, according to simulations (symbols) and to the analytical estimation derived from BKS theory [63] (see text).

\section{B. Climb process}

To verify how the dislocation climb proceeds at the atomic scale, we introduced vacancies in the simulation cell one by one, and the relaxation procedure was repeated. For each 
(a)

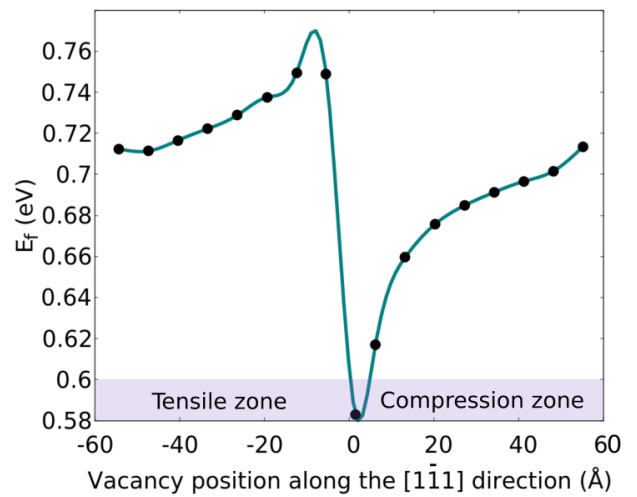

(b)

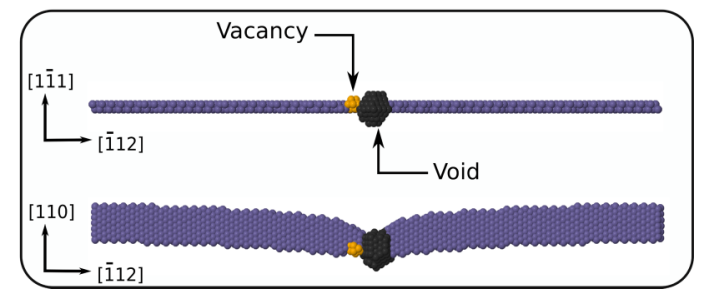

FIG. 3. (a) Formation energy of a vacancy against the distance in [111] direction to the dislocation anchored by a vacancy cluster (which radius is $r=6 \AA$ ). The applied shear stress is $\tau=40 \mathrm{MPa}$. (b) Atomic configuration corresponding to the minimal formation energy for the process described in (a). The color code is the same as in Fig. 1(a).

addition of a vacancy, we chose the atom to be removed according to an energy criterion which states that the vacancy is located where its formation is most favorable, i.e., where the formation energy is the smallest. The formation energy is computed for every atomic position in the simulation cell before we introduce a new vacancy. A kinetic Monte Carlo simulation as those proposed in Refs. [68,69] would be more appropriate to capture the time scale and the configuration entropy. Simulations with the KMC algorithm would also allow us to treat the dislocation mobility in the three-dimensional distribution of various obstacles. Here we need to determine only the series of the more favorable configurations in order to sketch out an average evolution of the system. We also neglected the contributions from crystal vibrations.

We computed the formation energy $E_{f}$ of a vacancy in the vicinity of a dislocation anchored by a cluster as follows:

$$
E_{f}=E_{(\mathrm{cell}+d+c+v)}-\frac{N_{\mathrm{at}}-1}{N_{\mathrm{at}}} E_{(\mathrm{cell}+d+c)},
$$

where $E_{(\text {cell }+d+c)}$ stands for the potential energy of the simulation cell including a dislocation and a vacancy cluster and $E_{(\text {cell }+d+c+v)}$ is the same but with one single vacancy in addition (Fig. 3). The number of atoms in the former is denoted by $N_{\text {at }}$. The formation energy $E_{f}$ in the compression zone is lower than in the tensile zone (see Fig. 3), as expected from the standard theory of dislocation [70]. At the $\mathrm{Z}$ coordinate where $E_{f}$ is minimum, i.e., in the $\{111\}$ plane contiguous to the glide plane located in the compression zone, we tested all the atomic positions. We noticed that the formation energy (a)

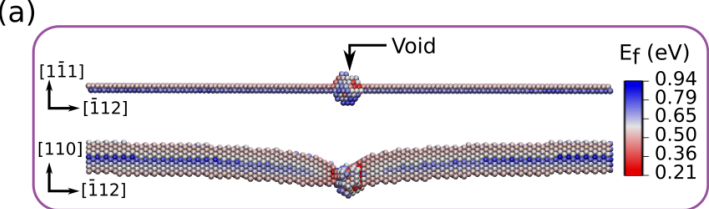

(b)

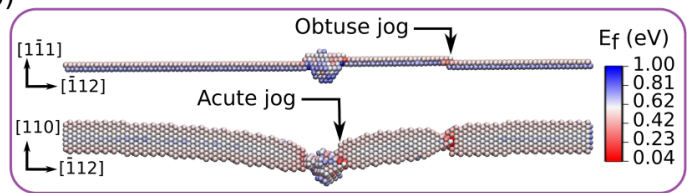

(c)

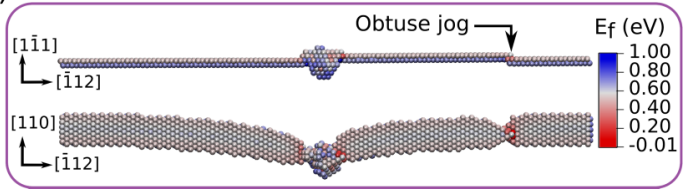

FIG. 4. Snapshot of our simulations along the introduction of vacancies one after the other (see text): (a) no additional vacancy, (b) after addition of 30 vacancies, and (c) after addition of 45 vacancies. The atom colors are fixed according to the formation energy of the vacancy which could be located at the position of the corresponding atom.

reaches a minimum on the trailing partial in the vicinity of the void [see Figs. 3(b) and 4(a)].

When adding more vacancies, we found that the adsorption of vacancies occurs essentially in the first-neighbor positions to the primary vacancy. After a certain number of adsorptions, it yields a pair of jogs [see Figs. 4(b) and 4(c)]. The subsequent adsorption is always more favorable at the bottom of a jog such that the step formed by the jog moves one lattice distance after each vacancy adsorption. We found that such a propagation is more favorable on the side of the obtuse jog. The difference between the formation energy on the obtuse jog and on the acute jog [35] is $0.2 \mathrm{eV}$ in favor of the former. Such asymmetry between jogs due to the presence of the void is expected to have barely no consequences on the velocity of the climb process. However, we admit that a study devoted to such an asymmetry would be useful in various fcc crystals.

\section{Elastic line model}

In order to compute the average distance over which a dislocation may glide through a random distribution of obstacles, we employed a 2D elastic line model [30-34]. It allowed us to consider realistic dislocation length and glide distances, not feasible directly in atomic-scale simulations. The dislocation line is discretized in segments of length $b$ and the position of each segment labeled by $i$ is determined by its coordinate $x_{i}$ along the line direction. For the sake of simplicity, an attractive potential $W(x)$ was used to model the dislocation-obstacle interaction, computed in atomic scale simulations. We chose the simple form $W(x)=-A\left(x^{2}-w^{2}\right)^{4} / w^{8}$, where $w$ is the range of the interaction beyond which the potential is zero, $x$ is the distance between the dislocation and the obstacle, and $A$ is the amplitude of the potential. The latter was fixed according to the pinning force [Eq. (3)], which means that we adjusted the parameters of the potential $W(x)$ such that $f_{p}$ is 
the extrema of $d W(x) / d x$, obtained at $x=w / \sqrt{7}$. It yields $A=343 \sqrt{7} w f_{p} / 1728$. The interaction range in $X$ direction, denoted by $w$ was fixed to $w=5 b$, such that we neglected the long-range elastic interactions. We assumed that the interaction potential is limited in the direction of the elastic line to only one segment of width $b$, which proved sufficient in different situations [34]. Therefore the total force on the segment $i$ is

$$
F_{i}=-\sum_{j} W^{\prime}\left(\left|x_{i}-X_{i, j}\right|\right)-\tau b^{2}-\Gamma\left(x_{i+1}+x_{i-1}-2 x_{i}\right) / b,
$$

where $X_{i, j}$ corresponds to the position of the obstacle ranked by $j$ along the $\mathrm{X}$ direction situated in front of segment $i$. The second term in Eq. (5) corresponds to the Peach-Koehler force on a segment of length $b$, while the third one represents the line tension contribution, which we determined as follows. Setting the same length for the elastic line and the dislocation in the atomic scale MS simulations, we applied the same shear stress in both simulations after having introduced a single obstacle whose the pinning force was sufficient to anchor the dislocation. Then we merely adjusted the coefficient $\Gamma$ such that we obtain similar curvature for the dislocation and the elastic line. Since the dislocation is dissociated in two partial dislocations in the atomic scale MS simulations, we have compared the elastic line profile to the average profile between the two partial dislocations. A more complete elastic line model allows to capture thoroughly the properties of the dissociated dislocation [34]. The elastic line profile is superimposed to the MS computation for the dislocation profile in Fig. 5(a). Through such a procedure we found for the edge dislocation in $\mathrm{Al}, \Gamma=3.0 \mathrm{eV} / \mathrm{nm}$. For comparison, the elastic theory predicts $\Gamma_{\mathrm{el}}=\mu_{0} b^{2} \frac{1-2 v}{4 \pi(1-v)} \ln (R / b)$ [71], where $R$ is the distance between the dislocation and its first periodic images, which corresponds roughly to the box size in the direction $\mathrm{Y}$ [112], $L_{y}=104 b$. It gives numerically $\Gamma_{\mathrm{el}}=2.7 \mathrm{eV} / \mathrm{nm}$.

For a dislocation of length $L$ in interaction with a single obstacle situated at $X_{n, 1}$, the sum of the forces in Eq. (5) at equilibrium leads to $\tau b L=-W^{\prime}\left(\left|x_{n}-X_{n, 1}\right|\right)$. It reaches a maximum when $\left|x_{n}-X_{n, 1}\right|=w / \sqrt{7}$ and thus when $\tau b L=f_{p}$. The equilibrium configuration of the elastic line is computed through a simple gradient algorithm where the gradient is given by Eq. (5). A random distribution of obstacles is constructed in the glide plane of the elastic line. Each obstacle is associated to a pinning force $f_{p}$ and the probability to find an obstacle on a given site of the plane is fixed by $c$, which we have identified to the probability of finding a cluster on a site of the crystal, which gives $c=n_{s} V_{\text {at }}$, where $n_{s}$ is the number of clusters per volume unit and $V_{\text {at }}$ is the atomic volume in the crystal. $V_{\text {at }}=$ $1.66 \times 10^{-29} \mathrm{~m}^{3}$ in Al crystal. Different random configurations were generated by changing the seed of the random number generator. Once the gradient algorithm has fulfilled the force criterion $\left(\left|f_{i}\right|<10^{-6} \mathrm{eV} / \mathrm{nm}\right)$, we assumed that the dislocation was at rest. Then, the dislocation is supposed to climb and circumvent the obstacles. We mimicked such a process by removing all the obstacles contiguous to the anchored elastic line. We are aware that the present model needs to be refined in terms of the process of dislocation unpinning as well as the dislocation interactions with clusters out of the glide plane. As it was shown in our atomic-scale computations (see (a)

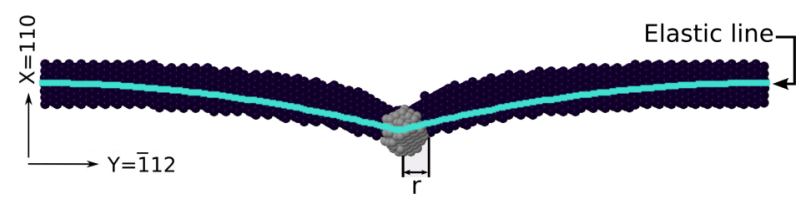

(b)

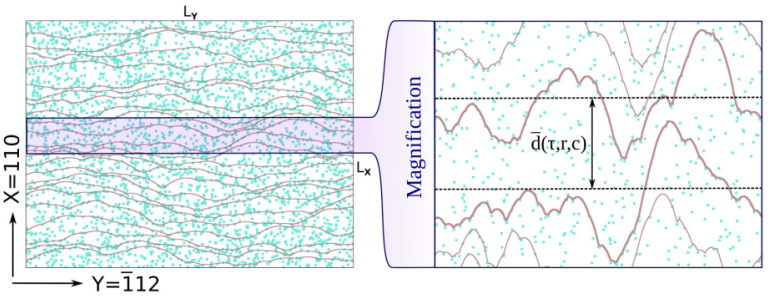

(c)

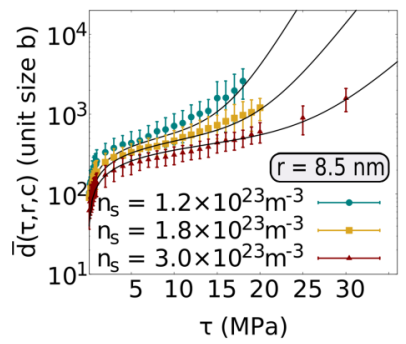

(d)

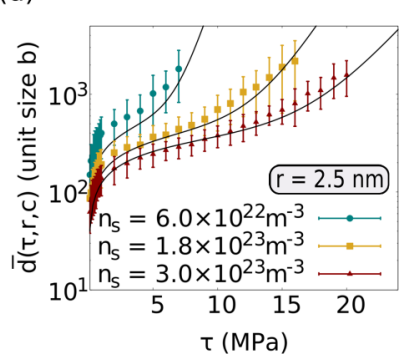

FIG. 5. (a) Profile of the elastic line (blue line) in interaction with an obstacle whose the pinning force $f_{p}$ corresponds to a radius $r=6.5 \AA$, under an applied shear stress $\tau=50 \mathrm{MPa}$. For comparison, we superimposed the profile of an edge dislocation in $\mathrm{Al}$, in interaction with a void of radius $r=6.5 \AA$, under the same shear stress. The color code is the same as in Fig. 1(a). The dislocation length and the elastic line have the same length $L=119 b$. (b) Snapshot of an elastic line (lines) anchored by a random distribution of obstacles (points). Each line corresponds to a different equilibrium configuration. The geometry of the glide plane is defined by $L_{\mathrm{X}}=L_{\mathrm{Y}}=1000 \mathrm{~b}$. The average distance between anchored configurations, $\bar{d}(\tau, r, c)$, is computed against the resolved shear stress $\tau$ for different obstacle concentration $c=n_{s} V_{\text {at }}$ : (c) with a pinning force $f_{p}(r=8.5 \mathrm{~nm})=8.5 \mathrm{eV} / \mathrm{nm}$ and (d) with a pinning force $f_{p}(r=2.5 \mathrm{~nm})=5.9 \mathrm{eV} / \mathrm{nm}$. The symbols are for simulation results and the lines correspond to a fit [see Eq. (6)].

Sec. II B), the jogs form in the vicinity of a void and propagate all along the dislocation length. The repetition of such a process allows to circumvent the obstacles of the anchored configuration. Eventually, it yields the dislocation unpinning, which supports our assumption in the elastic line model. For the present time, we are compelled to make assumptions that seem confirmed by the comparison, made later on, between our predictions and the experimental results. After the elastic line unpinning, the relaxation procedure was then repeated until the elastic line finds another anchored configuration [see Fig. 5(a)]. The difference between the initial and the final positions of the elastic line provides us an estimation of the jump distance for the dislocation. Repeating the computation for different random distributions allowed us to establish a thorough sampling for the jump distance and thus to compute its average $\bar{d}(\tau, r, c)$, against $\tau, c$, and $f_{p}$ [see Figs. 5(b) and $5(c)]$. Let us recall that thanks to the atomic scale computations, $f_{p}$ and the radius of the obstacle $r$ are unequivocally related [see Eq. (3)]. 
In Figs. 5(b) and 5(c), we reported the variation of $\bar{d}(\tau, r, c)$ against the applied stress for different pinning forces $f_{p}(r)$, i.e., for different obstacle sizes and for several concentrations. We introduced a phenomenological fit to reproduce the variation of $\bar{d}(\tau, r, c)$, which we computed from simulations:

$$
\begin{aligned}
\bar{d}(\tau, r, c)= & \sqrt{a_{0}(c) \tau}+\exp \left[a_{1}\left(c, f_{p}(r)\right) \tau\right] \\
& -a_{2}\left(c, f_{p}(r)\right) \tau-1,
\end{aligned}
$$

with

$$
\begin{aligned}
\mathrm{a}_{0}(\mathrm{c}) & =0.95 c^{-0.8}, \\
\mathrm{a}_{1}\left(\mathrm{c}, \mathrm{f}_{\mathrm{p}}(\mathrm{r})\right) & =2.19 \times 10^{-3} f_{p}(r)^{-1.4} c^{-0.62} \\
\mathrm{a}_{2}\left(\mathrm{c}, \mathrm{f}_{\mathrm{p}}(\mathrm{r})\right) & =9.91 f_{p}(r)^{-0.237} \exp (-1064 c),
\end{aligned}
$$

where the units are $b$ for $\bar{d}(\tau, r, c)$, MPa for the shear stress $\tau$, and $\mathrm{eV} / \mathrm{nm}$ for the pinning force $f_{p}$. When the resolved shear stress $\tau$ is much smaller than the critical shear stress, i.e., the stress limit above which the dislocation passes the obstacles with no resistance, the jump distance varies smoothly with stress [i.e., a square root variation according to our fit Eq. (6)]. By contrast, when $\tau$ approaches the limit of the critical stress, the jump distance increases at an exponential rate. Such a dramatic rise comes from the scarcity of configurations able to stop the dislocation at the approach of the critical stress.

\section{RESULTS}

The simulation method that we described in Sec. II allows us to determine the parameters needed for a climb assisted glide model. According to the Orowan law [72], the shear rate $\dot{\gamma}$ can be written as

$$
\dot{\gamma}=\rho b v,
$$

where $\rho$ is the density of mobile dislocations, which is assumed to follow the Taylor law [73-75] $\tau=0.3 \mu b \sqrt{\rho}, v$ is the dislocation velocity, and $b$ is the norm of the Burgers vector. The average time $t_{\text {climb }}$ required for the dislocation to climb over obstacles is much larger than the time required for the dislocation to glide between two anchored configurations. Therefore the dislocation velocity corresponds to the glide distance between two successive anchored configurations divided by $t_{\text {climb }}$. Hence $\dot{\gamma}$ can be written as

$$
\dot{\gamma}=\rho b \frac{\bar{d}\left(\tau, f_{p}(r), c\right)}{t_{\mathrm{climb}}},
$$

where $\bar{d}\left(\tau, f_{p}(r), c\right)$ is the average glide distance computed in Sec. IIC. The time required for a dislocation to climb over a distance equal to the average size of obstacles $r$ is merely $t_{\text {climb }}=r / v_{\text {climb }}$. The dislocation climb velocity $v_{\text {climb }}$ is estimated from the theory presented in Appendix B, which is derived in standard textbooks [70], such that

$$
t_{\text {climb }}=r / \frac{2 \pi D_{\text {bulk }}}{b \ln \left(R_{\infty} / r_{c}\right)}\left[\exp \left(\frac{V_{\text {at }} \sigma}{k_{\mathrm{B}} T}\right)-1\right],
$$

where $\sigma=\tau / S$ is the tensile stress, with $S$ as the Schmid factor, which takes usually the value of $1 / 2$ for geometrical reasons in polycrystals [53]. The radius $r_{c} \approx 2 b$ corresponds to the cutoff radius introduced in the elastic theory of dislocations [70], intending to represent the dislocation core. $V_{\text {at }}$ is the atomic

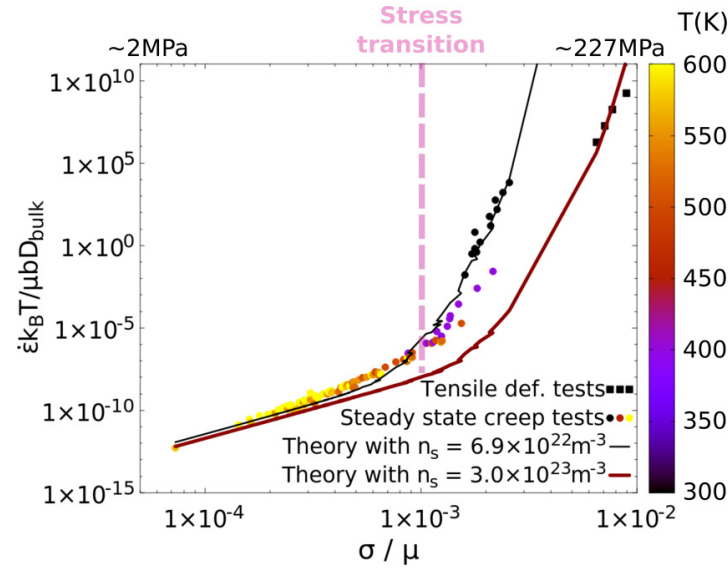

FIG. 6. Comparisons between the theoretical predictions (lines) and the experimental data (symbols) in Al: tensile tests [36] and creep tests [37-40]. The obstacle concentration $c$ in the theory was adjusted to fit the deformation experimental data. The symbols are colored according to the temperature of the corresponding experiment. The color code is reported on the right-hand side of the figure.

volume and $R_{\infty}$ stands for the average distance between dislocations, which is generally assumed to be given by $R_{\infty} \approx \frac{1}{\sqrt{\rho}}$. $k_{B}$ is the Boltzmann constant, $T$ is the temperature and $D_{\text {bulk }}$ is the bulk self-diffusion coefficient. The latter is computed via the law $D_{\text {bulk }}=D_{\text {bulk }}^{0} \exp \left(-Q_{\text {bulk }} / k_{B} T\right)$ with $D_{\text {bulk }}^{0}=$ $1.76 \times 10^{-5} \mathrm{~m}^{2} / \mathrm{s}$ and $Q_{\text {bulk }}=1.31 \mathrm{eV}$ (see Appendix A and Refs. [76-79]). Hence $\dot{\gamma}$ could be written as

$$
\dot{\gamma}=\rho \frac{\bar{d}\left(\tau, f_{p}(r), c\right)}{r} \frac{2 \pi D_{\text {bulk }}}{\ln \left(R_{\infty} / r_{c}\right)}\left[\exp \left(\frac{V_{\text {at }} \sigma}{k_{\mathrm{B}} T}\right)-1\right] .
$$

For the purpose of comparison with experiments, we remark that for the same geometrical reasons as for $\sigma=\tau / S$, the global strain rate is given by $\dot{\epsilon}=S \dot{\gamma}$. However, we have verified that such a geometrical factor has barely no consequences on the final computational results. The pinning force $f_{p}$ is related to the size of the vacancy cluster through Eq. (3), where the size of the clusters was fixed according to experimental observations by Dai et al. [6], i.e., $r=2.5 \mathrm{~nm}$ in single crystals of $\mathrm{Cu}$. Since we did not found any measures in $\mathrm{Al}$ for the cluster size, we assumed that it is similar in both materials. The average distance between anchored configurations $\bar{d}\left(\tau, f_{p}(r), c\right)$ was computed through the elastic line model (Sec. IIC). In the experimental observations by Dai et al. [6], it was found that for the small deformation $\gamma=0.05$ the number of clusters per volume unit was $n_{s}=1.1 \times 10^{22} \mathrm{~m}^{-3}$, while for the large deformation $\gamma=1.39$, the experimental observations indicate $n_{s}=6.9 \times 10^{22} \mathrm{~m}^{-3}$. Although the observations from Dai et al. were realized at room temperature in single crystals of $\mathrm{Cu}$, it seems reasonable to argue that the concentration of clusters increases also as the total strain in Al. In our computations, we therefore used two different values for the cluster concentration $c$, which we have adjusted according to the experimental strain. The results of our fits were reported in Fig. 6. We obtained a good agreement for the two types of experimental deformation tests. Concerning tensile deformation tests [36] where the maximum strain was $\gamma>1$, we found $c=5.0 \times 10^{-6}$, which corresponds to a number of clusters per volume unit 
$n_{s}=3.0 \times 10^{23} \mathrm{~m}^{-3}$. Concerning the creep deformation tests [37-40], where the maximum strain is much smaller [37], i.e., $\gamma<0.1$, we found that $c=1.15 \times 10^{-6}$, which corresponds to $n_{s}=6.9 \times 10^{22} \mathrm{~m}^{-3}$.

We emphasize that no parameter other than the concentration in the theory needs to be adjusted against experimental deformation tests. The experimental data were reported after normalization [51] by the diffusion coefficient $D_{\text {bulk }}$ in Fig. 6. The tensile stress is divided by the Al crystal shear modulus, whose temperature variation is fixed according to the law [65] $\mu=\mu_{0}\left(1+\frac{T_{m}}{\mu_{0}} \frac{d \mu}{d T} \frac{(T-300)}{T_{m}}\right)$, with Al melting temperature $T_{m}=$ $933 \mathrm{~K}$, the Al shear modulus at $300 \mathrm{~K}$ being $\mu_{0}=25.4 \times 10^{3}$ $\mathrm{MPa}$, and with $\frac{T_{m}}{\mu_{0}} \frac{d \mu}{d T}=-0.50$. These values were found in the same reference [65]. This normalized representation allows us to plot the experimental data over a broad range of temperatures and stresses. In Fig. 6, the experimental measurements form a well-marked hockey-stick curve where two regimes can be distinguished: (i) the intermediate stress level highlighted by Kassner in SSC of Al [28] where $\dot{\epsilon} \propto \sigma^{5}$; (ii) the high-stress regime, which some authors [51] interpreted as $\dot{\epsilon} \propto \sigma^{10}$. The fact that the experimental data fall on a same, well defined curve after being divided by the diffusion coefficient indicates that the self-diffusion process plays a key role in that regime of stress and temperature. In the present model as in many others $[27,45,47,48]$, this interplay between the self-diffusion and the deformation process is a reflection of the edge dislocation climb.

The model predictions show a satisfactory agreement with experimental deformation tests in regard of the fact that we avoided the adjustment of parameters except the concentration of nanovoids, which has not been measured in the different deformation tests. The characteristic hockey-stick curve formed by experimental data is well reproduced in the present theory. The transition between the two regimes of low and high stresses is well marked at $\sigma=10^{-3} \mu$ (see dashed line in Fig. 6) for both values of concentration. According to our computations, such a transition is essentially due to the variation of the dislocation glide distance. In Fig. 5, it appears that the average jump distance has clearly the behavior that corresponds to a hockey-stick curve.

\section{CONCLUSION}

Our simulations allowed us to quantify the interaction between dislocation and vacancy clusters and to determine the corresponding pinning forces for various cluster sizes and shapes. At very low stress, the dislocation is anchored on the cluster and can not pass the cluster through simple glide. In such a situation, we simulate the formation of jog that initiates the climb process. We show that the jog begins to form in the vicinity of the void and propagates along the line by absorption of vacancies. Then the elastic line model was used to account for the random distribution of the obstacles and to treat realistic geometries with respect to the dislocation glide distance and the dislocation length. Then we employed a classical theory for dislocation climb [47,70], which we supplemented with (i) atomic-scale simulations to describe the dislocation-cluster and dislocation-vacancy interactions and (ii) with an elastic-line model to compute the average glide distance of dislocations.

It is remarkable that we obtained a good agreement between the predictions drawn from such a simple glide assisted climb model with the two different sets of experimental data realized for stringently different strain rate. We admit that more experimental data, in particular about the vacancy cluster concentration, which was adjusted in the present study, would be required to make a conclusion. Along the strain rate curves (see Fig. 6), our simulations predict a stress transition. The physical origin of such transitions may come from the scarcity of anchored configurations encountered as the stress approaches the critical stress for pure glide. Such a transition coincides with the experimental transition observed in SSC experiments, which can be interpreted in a phenomenological manner as an abrupt change of the stress exponent in Norton's law (see for instance Ref. [45]). We show that a quantitative agreement between that theory and the experimental data is possible. We believe that this approach could be supported by direct simulations for dislocation climb $[68,80]$ that would permit to treat both climb and glide of dislocations on equal footing. In particular, we think that it is important to take into account (i) the interaction of the dislocation with a threedimensional distribution of obstacles and (ii) the competition between the thermal activation of glide on the dislocation climb, which could contribute to the kinetics of strain.

\section{ACKNOWLEDGMENTS}

We gratefully acknowledge enlightening discussions with Emmanuel Clouet [80]. This work has been carried out within the framework of the EUROfusion Consortium and has received funding from the Euratom research and training program 2014-2018 under Grant Agreement No. 633053. The views and opinions expressed herein do not necessarily reflect those of the European Commission.

\section{APPENDIX A: EXPERIMENTAL DATA FOR BULK SELF-DIFFUSION COEFFICIENT}

Experimental data concerning the bulk self-diffusion coefficients measured in $\mathrm{Al}$ [76-79] are reported in Fig. 7. To model $D_{\text {bulk }}$ as a function of the temperature, we have used the typical Arrhenius equation [65]

$$
D_{\text {bulk }}=D_{\text {bulk }}^{0} \exp \left(-Q_{\text {bulk }} / k_{B} T\right) \text {. }
$$

The bulk diffusion parameters are $D_{\text {bulk }}^{0}=1.76 \times 10^{-5} \mathrm{~m}^{2} / \mathrm{s}$ and $Q_{\text {bulk }}=1.31 \mathrm{eV}$. We have challenged this model with the experimental measures of different authors (see Fig. 7). The model agrees well with experiments in the range of temperature used in Fig. 6.

\section{APPENDIX B: THEORETICAL DEMONSTRATION OF THE CLIMB VELOCITY}

The derivation for the climb velocity of edge dislocations is guided by Hirth and Lothe's textbook [70]. The dislocation climb is determined by the flux of vacancies toward dislocation cores. According to the Einstein mobility relation, the drift velocity of vacancies is $v_{d}=D_{v} F / k_{B} T$, where $D_{v}$ stands 


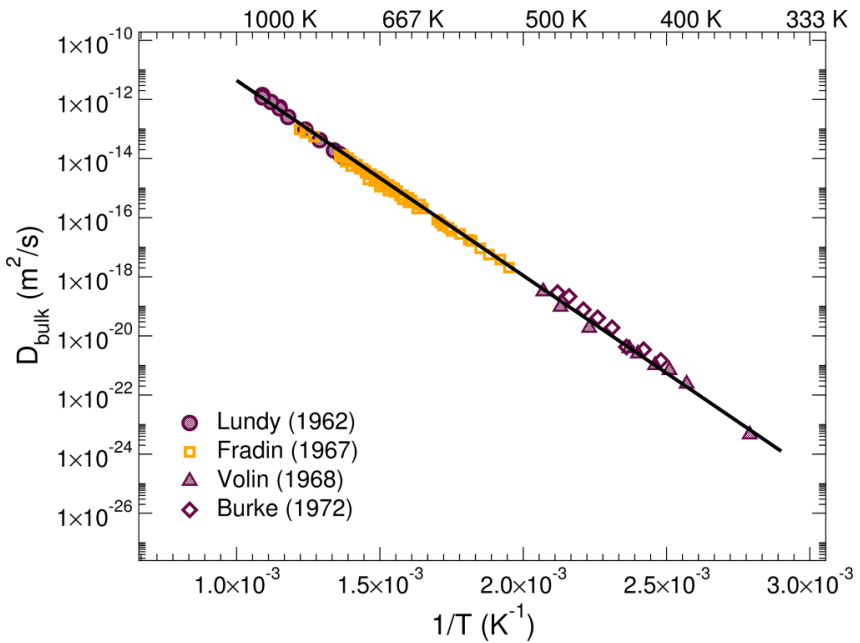

FIG. 7. Experimental data (symbols) for the bulk self-diffusion coefficient in Al [76-79] challenged with theoretical models (lines) (see text and Ref. [65]).

for the diffusivity and $F$ is a force field, which can be derived from the chemical potential of an ideal solution of vacancies: $\mu_{v}\left(C_{v}\right)=k_{B} T \ln C_{v} / C_{v}^{0}$, where $C_{v}^{0}$ is the vacancy concentration in the bulk at thermodynamical equilibrium. This expression accounts for the configurational entropy of a random distribution. The flux of vacancies along direction $\mathrm{X}$ is thus given by

$$
J_{x}=C_{v} v_{d}=-D_{v} d C_{v} / d x .
$$

At the steady state, the divergence of the flux is zero, which means with $C_{v}^{0}$ and $D_{v}$ constant in space, the Laplacian of $C_{v}$ is also null, which yields in cylindrical coordinates a solution of the form

$$
C_{v}=C_{v}^{0}+\delta C \frac{\ln \left(R_{\infty} / r\right)}{\ln \left(R_{\infty} / r_{c}\right)},
$$

where $R_{\infty}$ is the standard cutoff radius (usually taken equal to the half distance between dislocations), $r_{c}$ is the extension of the dislocation core, and $\delta C$ is a constant to be fixed. To obtain such a solution, it has been assumed that the vacancy concentration $C_{v}$ reaches its equilibrium value $C_{v}^{0}$ far from the dislocation cores, i.e., at $R_{\infty}$, and that inside the core, $C_{v}$ is a constant $C_{v}^{\text {core }}=C_{v}^{0}+\delta C$ determined by the competition between the osmotic force and the elastic one. The former is due to the creation or the annihilation of vacancies in the dislocation core while the latter is merely related to the effect of the applied stress. In the present coordinate [see Fig. 1(a)], the Peach-Koehler force perpendicular to the glide plane of an edge dislocation is $F_{z}^{\mathrm{el}}=\sigma_{x x} b L$, where $L$ represents the dislocation length. The osmotic force $F_{z}^{\text {osmo }}$ is due to the variation of the chemical potential $\mu_{v}$ when a row of vacancies is created along the dislocation line, which corresponds to a number of vacancies $L b h / V_{\text {at }}$, where $h$ stands for the distance the dislocation has moved. The total chemical potential variation is then $G=\mu_{v}\left(C_{v}^{\text {core }}\right) L b h / V_{\text {at }}$ from which the osmotic force can be easily derived against $h$ to obtain $F_{z}^{\text {osmo }}=-\mu_{v}\left(C_{v}^{\text {core }}\right) L b / v_{\text {at }}$, where we have noticed $C_{v}^{\text {core }}$, the vacancy concentration inside the region of the core, limited by $r_{c}$. At the steady state, the balance between the osmotic and the elastic forces allows us to equate the previous expressions, which gives $k_{B} T \ln C_{v}^{\text {core }} / C_{v}^{0}=\sigma_{x x} v_{\text {at }}$, which also means

$$
C_{v}^{\text {core }}=C_{v}^{0} e^{\frac{\sigma_{x x} v_{\text {at }}}{k_{B} T}} \text {. }
$$

Combining Eqs. (B2) and (B3), we deduce that $\delta C=C_{v}^{0}\left(e^{\frac{\sigma_{X X} v_{\text {at }}}{k_{B} T}}-1\right)$, which fully determines the concentration field for vacancies. Here, we do not take the linear approximation for this expression as in the textbook [81] since we aim to compute the creep rate over a broad stress range. The number of vacancies that move away from the dislocation core per unit of time is given by the integral of the flux $J$ [Eq. (B1)] on a cylindrical surface centered on the dislocation line with radius $r>r_{c}$, which is given by $I=-2 \pi r L D_{v} \partial C_{v} / \partial r$. Using Eq. (B2), we obtain $I=2 \pi L D_{v} \delta C / \ln \left(R_{\infty} / r_{c}\right)$. When a vacancy has been emitted, the distance the dislocation core has climbed is roughly $v_{\text {at }} / b / L$, the elementary crystal dimension perpendicular to the glide plane, which allows us to write the climb velocity $v_{\text {climb }}=I v_{\text {at }} / b / L$ and to obtain

$$
v_{\text {climb }}=\frac{2 \pi D_{\text {bulk }}\left(e^{\frac{\sigma_{X x} v_{\text {at }}}{k_{B} T}}-1\right)}{b \ln \left(R_{\infty} / r_{c}\right)},
$$

where $D_{\text {bulk }}=v_{\text {at }} C_{v}^{0} D_{v}$ corresponds to the self-diffusion coefficient. In our study, we consider that $\sigma_{x x}$ is roughly given by the tensile stress $\sigma$, noting that in principle the relation between both components is linear.
[1] M. Kiritani, Similarity and difference between fcc, bcc and hcp metals from the view point of point defect cluster formation, J. Nucl. Mater 276, 41 (2000).

[2] M. Kiritani, Story of stacking fault tetrahedra, Mater. Chem. Phys. 50, 133 (1997).

[3] S. Kojima, Y. Satoh, H. Taoka, I. Ishida, T. Yoshiie, and M. Kiritani, Confirmation of vacancy-type stacking fault tetrahedra in quenched, deformed and irradiated face-centred cubic metals, Philos. Mag. A 59, 519 (1989).

[4] M. H. Loretto, L. M. Clarebrough, and R. L. Segall, Stackingfault tetrahedra in deformed face-centred cubic metals, Philos. Mag. 11, 459 (1965).
[5] L. M. Clarebrough, P. Humble, and M. H. Loretto, Voids in quenched copper, silver and gold, Acta Metall. 15, 1007 (1967).

[6] Y. Dai and M. Victoria, Defect structures in deformed fcc metals, Acta Mater. 45, 3495 (1997).

[7] H. Wang, D. Xu, R. Yang, and P. Veyssière, The transformation of edge dislocation dipoles in aluminium, Acta Mater. 56, 4608 (2008).

[8] H. Wang, D. S. Xu, R. Yang, and P. Veyssière, The formation of stacking fault tetrahedra in $\mathrm{Al}$ and $\mathrm{Cu}$ : I. Dipole annihilation and the nucleation stage, Acta Mater. 59, 1 (2011).

[9] H. Wang, D. S. Xu, R. Yang, and P. Veyssiere, The formation of stacking fault tetrahedra in $\mathrm{Al}$ and $\mathrm{Cu}$ : II. SFT growth 
by successive absorption of vacancies generated by dipole annihilation, Acta Mater. 59, 10 (2011).

[10] H. Wang, D. Rodney, D. Xu, R. Yang, and P. Veyssière, Pentavacancy as the key nucleus for vacancy clustering in aluminum, Phys. Rev. B 84, 220103 (2011).

[11] H. Wang, D. S. Xu, P. Veyssière, and R. Yang, Interstitial loop strengthening upon deformation in aluminum via molecular dynamics simulations, Acta Mater. 61, 3499 (2013).

[12] S. Brinckmann, R. Sivanesapillai, and A. Hartmaier, On the formation of vacancies by edge dislocation dipole annihilation in fatigued copper, Int. J. Fract. 33, 1369 (2011).

[13] G. Saada, Interaction de dislocations érouissage et production de défauts ponctuels dans les métaux c.f.c, Acta Metall. 9, 166 (1961).

[14] T. Hatano and H. Matsui, Molecular dynamics investigation of dislocation pinning by a nanovoid in copper, Phys. Rev. B 72, 094105 (2005).

[15] D. J. Bacon and Yu. N. Osetsky, The atomic-scale modeling of dislocation-obstacle interactions in irradiated metals, JOM 59, 40 (2007).

[16] M. Bahramyan, R. T. Mousavian, and D. Brabazon, Molecular dynamic simulation of edge dislocation-void interaction in pure $\mathrm{Al}$ and Al-Mg alloy, Mater. Sci. Eng. A 674, 82 (2016).

[17] B. D. Wirth, V. V. Bulatov, and T. D. de la Rubia, Dislocationstacking fault tetrahedron interactions in $\mathrm{Cu}$, J. Eng. Mater. Technol. 124, 329 (2002).

[18] J. S. Robach, I. M. Robertson, H.-J. Lee, and B. D. Wirth, Dynamic observations and atomistic simulations of dislocationdefect interactions in rapidly quenched copper and gold, Acta Mater. 54, 1679 (2006).

[19] K. Tougou, K. Nogiwa, A. Shikata, and K.-i. Fukumoto, In-situ tem observation of dislocation interaction with cavity in ionirradiated pure vanadium during tensile test, Mater. Trans. 54, 1095 (2013).

[20] D. J. Bacon, Yu. N. Osetsky, and D. Rodney, Dislocationobstacle interactions at the atomic level, Dislocations Solids 15, 1 (2009).

[21] S. M. Keralavarma, T. Cagin, A. Arsenlis, and A. A. Benzerga, Power-Law Creep from Discrete Dislocation Dynamics, Phys. Rev. Lett. 109, 265504 (2012).

[22] F. Boioli, P. Carrez, P. Cordier, B. Devincre, and M. Marquille, Modeling the creep properties of olivine by 2.5-dimensional dislocation dynamics simulations, Phys. Rev. B 92, 014115 (2015).

[23] R. W. Balluffi and D. N. Seidman, Diffusion-limited climb rate of a dislocation: Effect of climb motion on climb rate, J. Appl. Phys. 36, 2708 (1965).

[24] R. W. Balluffi, Mechanisms of dislocation climb, Phys. Stat. Sol. 31, 443 (1969).

[25] J. Grilhe, M. Boisson, K. Seshan, and E. J. Gaboriaud, Climb model of extended dislocations in fcc metals, Philos. Mag. 36, 923 (1977).

[26] S. Sarkar, Ju Li, W. T. Cox, E. Bitzek, T. J. Lenosky, and Y. Wang, Finding activation pathway of coupled displacive-diffusional defect processes in atomistics: Dislocation climb in fcc copper, Phys. Rev. B 86, 014115 (2012).

[27] J. Weertman, Dislocation climb theory of steady-state creep, ASM. Trans. Quart. 61, 681 (1968).

[28] M. E. Kassner and M.-T. Pérez-Prado, Five-power-law creep in single phase metals and alloys, Prog. Mater. Sci. 45, 1 (2000).
[29] H. Mecking and Y. Estrin, The effect of vacancy generation on plastic deformation, Script. Metall. 14, 815 (1980).

[30] A. J. E. Foreman and M. J. Makin, Dislocation movement through random arrays of obstacles, Philos. Mag. 14, 911 (1966).

[31] J. W. Morris, Jr. and D. H. Klahn, Thermally activated dislocation glide through a random array of point obstacles: Computer simulation, J. Appl. Phys. 45, 2027 (1974).

[32] K. Hanson and J. W. Morris, Jr., Limiting configuration in dislocation glide through a random array of point obstacles, J. Appl. Phys. 46, 983 (1975).

[33] A. I. Landau, Thermally activated motion of a dislocation through a random array of point obstacles, Phys. Stat. Sol. A 30, 659 (1975)

[34] L. Proville and S. Patinet, Atomic-scale models for hardening in fcc solid solutions, Phys. Rev. B 82, 054115 (2010).

[35] P. B. Hirsch, Extended jogs in dislocations in face-centred cubic metals, Philos. Mag. 7, 67 (1962).

[36] A. Airod, H. Vandekinderen, J. Barros, R. Colás, and Y. Houbaert, Constitutive equations for the room temperature deformation of commercial purity aluminum, J. Mater. Process. Technol. 134, 398 (2003).

[37] C. N. Ahlquist and W. D. Nix, The measurement of internal stresses during creep of $\mathrm{Al}$ and $\mathrm{Al}-\mathrm{Mg}$ alloys, Acta Metall. 19, 373 (1971).

[38] A. J. Ardell and S. S. Lee, A dislocation network theory of Harper-Dorn creep: I Steady state creep of monocrystalline Al, Acta Metall. 34, 2411 (1986).

[39] S. V. Raj, On the grain size dependence of Harper-Dorn creep, Mater. Sci. Eng. 96, 57 (1987).

[40] S. Straub and W. Blum, Does the "natural" third power law of steady state creep hold for pure aluminium? Script. Metall. Mater. 24, 1837 (1990).

[41] C. Herring, Diffusional viscosity of a polycrystalline solid, J. Appl. Phys. 21, 437 (1950).

[42] R. L. Coble, A model for boundary diffusion controlled creep in polycrystalline materials, J. Appl. Phys. 34, 1679 (1963).

[43] B. Burton, The low stress creep of aluminium near to the melting point: The influence of oxidation and substructural changes, Philos. Mag. 25, 645 (1972).

[44] J. P. Poirier, On the symmetrical role of cross-slip of screw dislocations and climb of edge dislocations as recovery processes controlling high-temperature creep, Rev. Phys. Appl. 11, 731 (1976).

[45] J. P. Poirier, Is power-law creep diffusion-controlled? Acta Metall. 26, 629 (1978).

[46] C. R. Barrett and W. D. Nix, A model for steady state creep based on the motion of jogged screw dislocations, Acta Metall. 13, 1247 (1965).

[47] W. Blum, Role of dislocation annihilation during steady-state deformation, Phys. Stat. Sol. B 45, 561 (1971).

[48] W. Blum and H. Schmidt, Steady state and transient creep of al at $400 \mathrm{k}$ - an analysis in terms of recovery controlled by thermally activated glide, Res Mechanica 9, 105 (1983).

[49] H. E. Evans and G. Knowles, A model of creep in pure materials, Acta Metall. 25, 963 (1977).

[50] J.-P. Poirier, Creep of Crystals: High-Temperature Deformation Processes in Metals, Ceramics and Minerals (Cambridge University Press, Cambridge, 1985).

[51] M. E. Kassner, Fundamentals of Creep in Metals and Alloys (Butterworth-Heinemann, Oxford, 2015). 
[52] B. Chen, P. E. J. Flewitt, A. C. F. Cocks, and D. J. Smith, A review of the changes of internal state related to high temperature creep of polycrystalline metals and alloys, Int. Mater. Rev. 60, 1 (2015).

[53] M. Sauzay, Effet de l'anisotropie élastique cristalline sur la distribution des facteurs de schmid à la surface des polycristaux, Comptes Rendus Mecanique 334, 353 (2006).

[54] L. Kubin, Dislocations, Mesoscale Simulations and Plastic Flow (Oxford University Press, Oxford, 2013).

[55] T. Hochrainer, M. Zaiser, and P. Gumbsch, A three-dimensional continuum theory of dislocation systems: Kinematics and meanfield formulation, Philos. Mag. 87, 1261 (2007).

[56] D. Rodney, L. Ventelon, E. Clouet, L. Pizzagalli, and F. Willaime, $\mathrm{Ab}$ initio modeling of dislocation core properties in metals and semiconductors, Acta Mater. 124, 633 (2017).

[57] X.-Y. Liu, P. P. Ohotnicky, J. B. Adams, C. L. Rohrer, and R. W. Hyland, Anisotropic surface segregation in $\mathrm{Al} \mathrm{Mg}$ alloys, Surf. Sci. 373, 357 (1997).

[58] F. Ercolessi and J. B. Adams, Interatomic potentials from firstprinciples calculations: the force-matching method, EPL 26, 583 (1994).

[59] E. Clouet, The vacancy-edge dislocation interaction in fcc metals: A comparison between atomic simulations and elasticity theory, Acta Mater. 54, 3543 (2006).

[60] M. Landeiro Dos Reis, A. Choudhury, and L. Proville, Ubiquity of quantum zero-point fluctuations in dislocation glide, Phys. Rev. B 95, 094103 (2017).

[61] J. Dérès, L. Proville, and M.-C. Marinica, Dislocation depinning from nano-sized irradiation defects in a bcc iron model, Acta Mater. 99, 99 (2015).

[62] Yu. Mishin, M. J. Mehl, D. A. Papaconstantopoulos, A. F. Voter, and J. D. Kress, Structural stability and lattice defects in copper: $A b$ initio, tight-binding, and embedded-atom calculations, Phys. Rev. B 63, 224106 (2001).

[63] D. J. Bacon, U. F. Kocks, and R. O. Scattergood, The effect of dislocation self-interaction on the Orowan stress, Philos. Mag. 28, 1241 (1973).

[64] R. O. Scattergood and D. J. Bacon, The Orowan mechanism in anisotropic crystals, Philos. Mag. 31, 179 (1975).

[65] H. J. Frost and M. F. Ashby, Deformation Mechanism Maps: The Plasticity and Creep of Metals and Ceramics (Pergamon Press, Oxford, 1982).
[66] G. Henkelman, G. Jóhannesson, and H. Jónsson, Progress on Theoretical Chemistry and Physics (Kluwer, Dordrecht, 2000), pp. 269-300.

[67] G. Henkelman, G. Jóhannesson, and H. Jónsson, Methods for finding saddle points and minimum energy paths: Theoretical methods in condensed phase chemistry, in Progress in Theoretical Chemistry and Physics, edited by S. D. Schwartz (Springer, Dordrecht, 2000), Vol. 5, Chap. 10, pp. 269-302.

[68] M. Kabir, T. T. Lau, D. Rodney, S. Yip, and K. J. Van Vliet, Predicting Dislocation Climb and Creep from Explicit Atomistic Details, Phys. Rev. Lett. 105, 095501 (2010).

[69] T. T. Lau, X. Lin, S. Yip, and K. J. Van Vliet, Atomistic examination of the unit processes and vacancy-dislocation interaction in dislocation climb, Script. Mater. 60, 399 (2009).

[70] J. P. Hirth and J. Lothe, Theory of Dislocations (Wiley, New York, 1982), p. 555.

[71] J. P. Hirth and J. Lothe, Theory of Dislocations (Wiley, New York, 1982), p. 180.

[72] E. Orowan, Problems of plastic gliding, Proc. Phys. Soc. 52, 8 (1940).

[73] M. E. Kassner and M. E. McMahon, The dislocation microstructure of aluminium, Metall. Trans. 18, 835 (1987).

[74] M. E. Kassner, Taylor hardening in five-power-law creep of metals and class M alloys, Acta Mater. 52, 1 (2004).

[75] M. E. Kassner, P. Kumar, and W. Blum, Harper-dorn creep, Int. J. Plast. 23, 980 (2007).

[76] T. S. Lundy and J. F. Murdock, Diffusion of $\mathrm{Al}^{26}$ and $\mathrm{Mn}^{54}$ in aluminum, J. Appl. Phys. 33, 1671 (1962).

[77] F. Y. Fradin and T. J. Rowland, NMR measurement of the diffusion coefficient of pure aluminum, Appl. Phys. Lett. 11, 207 (1967).

[78] T. E. Volin and R. W. Balluffi, Annealing kinetics of voids and the self-diffusion coefficient in aluminum, Phys. Stat. Sol. B 25, 163 (1968).

[79] J. Burke and T. R. Ramachandran, Self-diffusion in aluminum at low temperatures, Metall. Trans. 3, 147 (1972).

[80] E. Clouet, Predicting dislocation climb: Classical modeling versus atomistic simulations, Phys. Rev. B 84, 092106 (2011).

[81] J. P. Hirth and J. Lothe, Theory of Dislocations (Wiley, New York, 1982), p. 315. 